\title{
A novel COL6A2 mutation causing late-onset limb-girdle muscular dystrophy
}

\author{
Manu Jokela ${ }^{1,2}$ D $\cdot$ Sara Lehtinen ${ }^{3} \cdot$ Johanna Palmio $^{1} \cdot$ Anna-Maija Saukkonen ${ }^{4}$ Sanna Huovinen ${ }^{5} \cdot$ Anna Vihola $^{3,6}$. \\ Bjarne Udd ${ }^{1,6,7}$
}

Received: 1 December 2018 / Revised: 21 March 2019 / Accepted: 1 April 2019 / Published online: 8 April 2019

(c) The Author(s) 2019

\begin{abstract}
Limb-girdle muscular dystrophies (LGMD) are genetic disorders characterized by weakness of predominantly proximal limb and trunk muscles due to progressive loss of muscle tissue. Collagen VI-related muscular dystrophies usually display more generalized muscle involvement combined with contractures and/or hyperlaxity of distal finger joints. LGMD-like phenotype of collagenopathy has only rarely been described and as reported is usually of childhood onset. We identified a Finnish family with COL6A2-related LGMD with autosomal dominant inheritance and very late onset at 40-60 years of age. Since the mutation was previously unreported, the pathognomonic findings on muscle MRI were the decisive clue for the correct diagnosis.
\end{abstract}

Keywords Myopathy $\cdot$ Collagenopathy $\cdot$ Limb-girdle muscular dystrophy $\cdot$ COL6A2

\section{Introduction}

Limb-girdle muscular dystrophies (LGMD) are genetic disorders characterized by progressive weakness and loss of muscle tissue predominantly affecting proximal limb

Manu Jokela

mejoke@utu.fi

1 Neuromuscular Research Center, Department of Neurology, Tampere University and University Hospital, Tampere, Finland

2 Division of Clinical Neurosciences, Turku University Hospital, University of Turku, Kiinamyllynkatu 4-8, 20520 Turku, Finland

3 Neuromuscular Research Center, Fimlab Laboratories, Tampere University and University Hospital, Tampere, Finland

4 Department of Neurology, Northern Karelia Central Hospital, Joensuu, Finland

5 Department of Pathology, Fimlab Laboratories, Tampere University Hospital, Tampere, Finland

6 Department of Medical Genetics, Folkhälsan Institute of Genetics, Haartman Institute, University of Helsinki, Helsinki, Finland

7 Department of Neurology, Vasa Central Hospital, Vasa, Finland muscles. The degeneration of muscles is detectable both on muscle MRI and biopsy, which results in end-stage pathology in the severely affected muscles. LGMDs are further subdivided into autosomal dominant and autosomal recessive forms. The growing number and increasing heterogeneity of different LGMD forms reported over the years prompted a recent European Neuromuscular Center (ENMC) workshop to suggest revisions for the definition and classification of LGMD [1].

Muscle collagenopathies are caused by mutations in COL6A1, COL6A2 or COL6A3 genes, which encode different alpha-subunits of fibroblast-secreted collagen VI fibrils deposited in the extracellular matrix. Collagen VI is required for the physical integrity and cohesion of muscle tissue as well as for signaling functions. The phenotype of collagenopathies usually includes a combination of contractures with progressive muscle weakness and wasting of variable severity [2]. An LGMD-like phenotype without significant contractures has been reported only rarely [3].

We describe a Finnish family with COL6A2-related dominant LGMD and very late onset (40-60 years). An unreported, unique mutation in the COL6A2 gene (c.2107A $>\mathrm{C}$ p.T703P, according to the transcript NM_001849) was identified in the proband using our targeted next-generation sequencing assay of myopathy-associated genes (MyoCap) 
[4]. Sanger sequencing confirmed correct segregation of the detected mutation in three other family members.

\section{Materials and methods}

\section{Patients}

Four members of a Finnish family with late-onset, autosomal dominant myopathy underwent clinical and laboratory, muscle imaging, histopathological and molecular genetics studies to identify the causative genetic defect. All patients provided informed consent for the examinations and the study was approved by the Tampere University Hospital ethics board.

\section{DNA sequencing}

DNA samples of the proband (II-2) and one of his affected sister's (II-1) were studied by targeted next-generation sequencing. The exons of 363 myopathy-associated genes were targeted using our custom MYOcap assay (Roche Nimblegen, Madison, WI, USA). The list of targeted genes is available upon request. Nimblegen SeqCap EZ Choice Library protocol was used for capture and target enrichment. The enriched library was sequenced by Illumina HiSeq 4000 Sequencher to 75 bp paired-end read length. The capture, enrichment and next-generation sequencing were performed at Oxford Genomics Centre (OGC at Wellcome Trust Centre for Human Genetics, Oxford, UK). The read/raw data were analyzed using an in-house developed pipeline [4].

Sanger sequencing of exon 26 of COL6A2 gene was used to analyze DNA samples of family members II-3 and II-4. The exon of interest was amplified by PCR $(2 \times$ PCR Master Mix; ThermoFisher Scientific, Waltham, MA, USA) and sequenced using Big-Dye Terminator v3.1 Kit on an ABI3130×l automatic Genetic Analyzer (Applied Biosystems, Foster City, CA, USA). Primer sequences are available upon request. Sequence analysis was performed with Sequencher 5.1 software (Gene Codes Corporation, Ann Arbor, MI).

\section{Muscle MRI}

MRI scans with axial sections of the lower limb muscles and using T1-weighted and short TI inversion recovery sequences were evaluated in four family members (II-1, II-2, II-3, II-4).

\section{Muscle histopathology and immunofluorescence studies}

Muscle biopsies were analyzed in patients II-1, II-2 and II-4, including hematoxylin and eosin (H\&E), Gomöri trichrome, NADH-TR, COX-SDH and neonatal myosin heavy chain (MyHC) stains. MyHC slow and MyHC fast double staining was also assessed. For the immunofluorescence studies, the primary antibodies used for immunofluorescent double staining were mouse monoclonal anti-collagen VI, clone VI-26 (Merck MAB3303) and rat monoclonal anti-merosin/ LAMA2, clone 4H8-2 (Abcam ab11576). Muscle biopsies [patients II-1 and II-2; healthy control free of muscle disease; and disease control, a patient with early-onset collagenopathy (COL6Al c.1056+1G >A p.G335_D352del)] were snap-frozen in liquid nitrogen-cooled isopentane, and $6 \mu \mathrm{m}$ muscle cryosections were prepared on SuperFrost + sides. The sections were fixed with 4\% PFA for $15 \mathrm{~min}$, and permeabilized using $0.05 \%$ Triton X-100 in PBS for $10 \mathrm{~min}$. After blocking with $2 \%$ BSA for $30 \mathrm{~min}$, the pooled primary antibody mix was added and incubated at $+8^{\circ} \mathrm{C}$ overnight. The following day, the slides were washed with PBS, and secondary antibody incubation was performed using Alexa546 and Alexa-488 conjugated antibodies, for $1 \mathrm{~h}$ at room temperature. An Axioplan 2 epifluorescence microscope (Carl Zeiss Microscopy $\mathrm{GmbH}$ ) was used for imaging.

\section{Results}

The proband (II-2) first noticed proximal weakness in the lower limbs when lifting heavy objects or walking up stairs at the age of 59-60 years. Previously he had been athletic and in his forties he was still able to ski for $10 \mathrm{~km}$. After age 62 , he had also experienced weakness in proximal upper limbs. On examination at age 66 , he had proximal weakness in the upper and lower limbs of MRC grade 3-4, slight scapular winging and distal weakness in the lower limbs (Table 1). EMG/NCS and muscle biopsy (Fig. 1, Table 1) showed abnormalities compatible with dystrophy, but also signs of neurogenic involvement most likely related to previous $L 4$ radiculopathies.

His two sisters (II-1, II-3) also had a similar phenotype characterized by proximal muscle weakness from ages 40 and 50 years onwards, respectively. Lower limb muscle MRI showed layers of fatty replacement in a "tigroid" pattern (Fig. 2a, c and e). Muscle biopsies showed myopathic features including fiber atrophy, increased internal nuclei and mild fibrofatty tissue replacement (Table 1, Fig. 1). None of the patients had significant joint contractures, although range of motion in ankle dorsiflexion was limited due to mildly tight heel cords. 


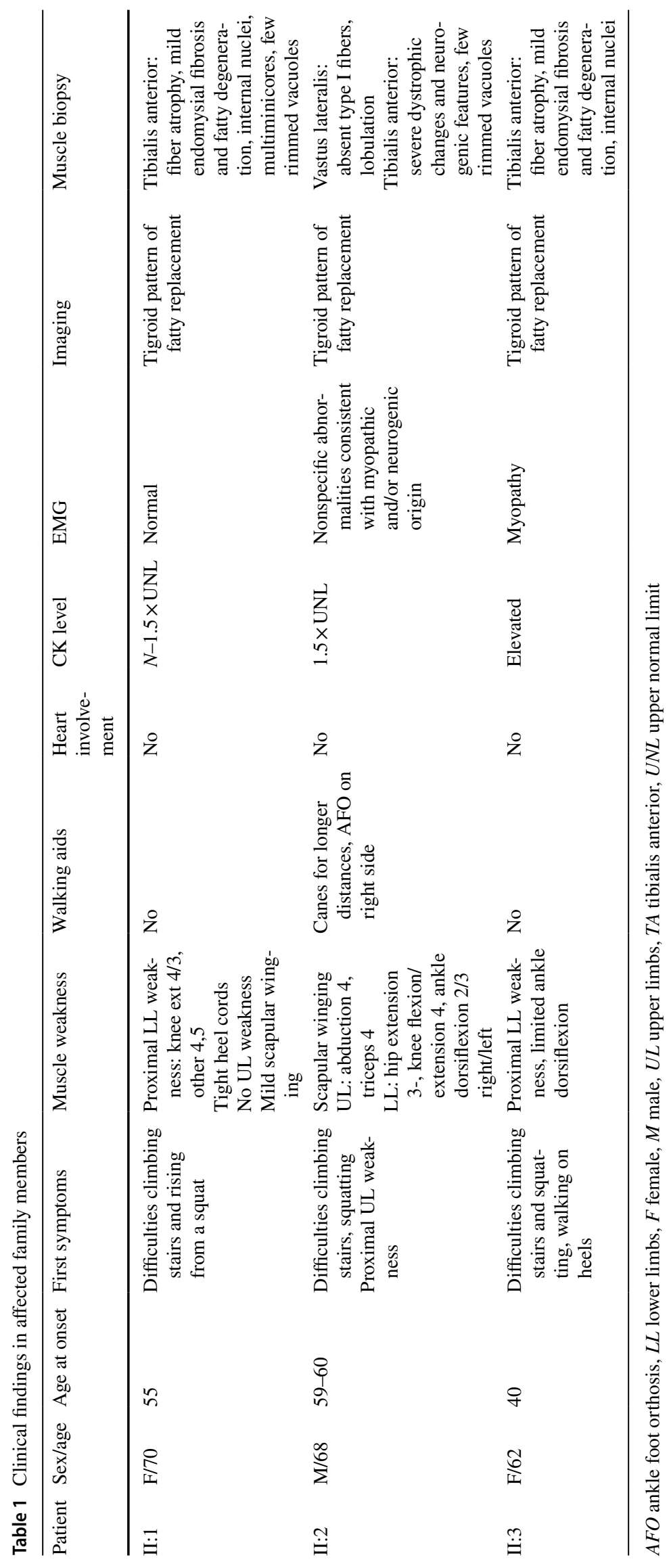


Fig. 1 a Pedigree of the family. Solid black symbols represent clinically affected individuals. Individual I-2 labeled solid gray has not been clinically evaluated but was reportedly affected. DNA sample was available from individuals marked with a dot. b-d Pathological changes observed in tibialis anterior muscle biopsies (Haematoxylin andEosin stain). Muscle biopsy from the proband (II-2) shows dystrophic features including many internalized nuclei, fiber size variation, fiber splitting and increase of connective and adipose tissue. Small group atrophy signifying neurogenic involvement is also present, probably related to an additional radiculopathy in this patient. Biopsies obtained from the proband's sisters (II-1, II-3; c, d, respectively) showed fiber atrophy, internal nuclei and endomysial fibrofatty degeneration

The patients were confirmed to carry a heterozygous c.2107A $>$ C p.T703P mutation in the COL6A2 gene (pedigree in Fig. 1). Targeted next-generation sequencing specified seven rare variants $(\mathrm{MAF}<0.01)$ shared between proband and his affected sister II-1. Of these, only c. $2107 \mathrm{~A}>\mathrm{C}$ in COL6A2 gene was unique and, therefore, considered likely pathogenic. This was supported by in silico pathogenicity prediction programs (Polyphen, Sift, Provean, Mutation Taster), which all predicted the variant to be damaging. Missense or synonymous variants in OBSCN, PLEC, PLEKHG4, NEB, ITGA7 were excluded on the basis that they were too common (MAF $>0.001$ ) for a fully penetrant dominant mutation and because they were classified as benign/tolerated using in silico pathogenicity assessment tools. Furthermore, one of the variants was in a gene that is not expressed in skeletal muscle (MYLK3). The same COL6A2 mutation was detected by Sanger sequencing also in the affected sister II-3. One of the sisters (II-4) had minor myalgic complaints at age 60 years and was, therefore, also studied by muscle MRI, which showed normal findings (Fig. 2). She was found not to carry the COL6A2 variant that was detected in the affected family members.

We did not observe clear abnormality in the immunofluorescent double staining of COLVI and merosin/LAMA2 in the muscle biopsies from patients II- 1 and II-2, indicating that there is at least partial co-localization of these proteins, visualized as mostly yellow overlapping labeling in the muscle fiber basal lamina (Fig. 3c, d).

\section{Discussion}

We describe a family with three patients having an LGMDlike phenotype of collagenopathy with atypical late onset. Symptoms in Bethlem myopathy (OMIM \#158,810) usually start in childhood with the majority of patients requiring mobility aids after 50 years of age [5].

Although distal lower limb weakness was present at the later disease stages in our patients, the initial symptoms in all our patients were due to proximal muscle weakness. A unique variant in COL6A2 (c. $2107 \mathrm{~A}>\mathrm{C}$ p.T703P) was
A
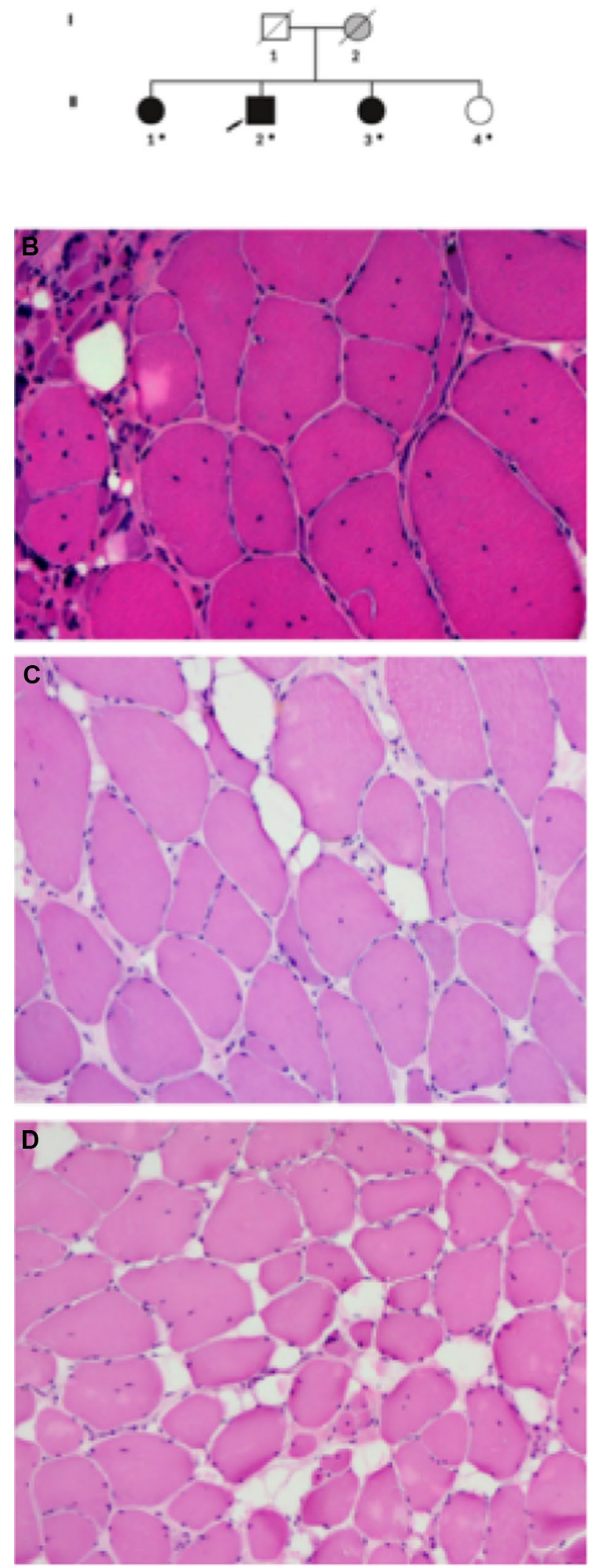


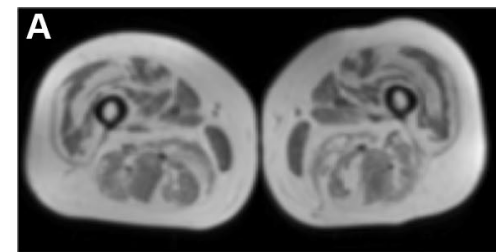

B

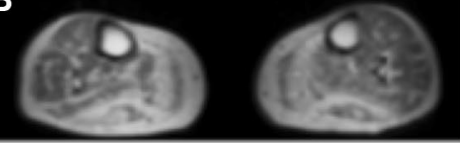

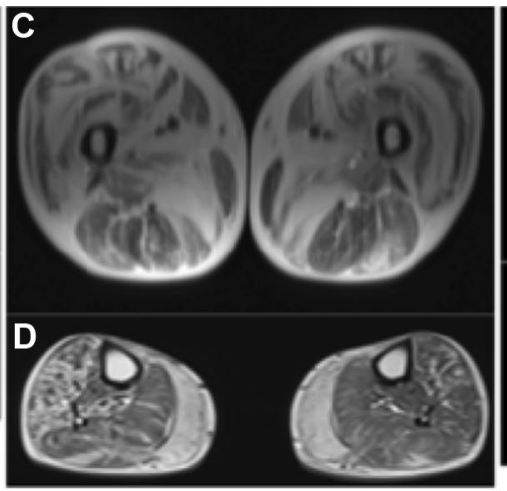
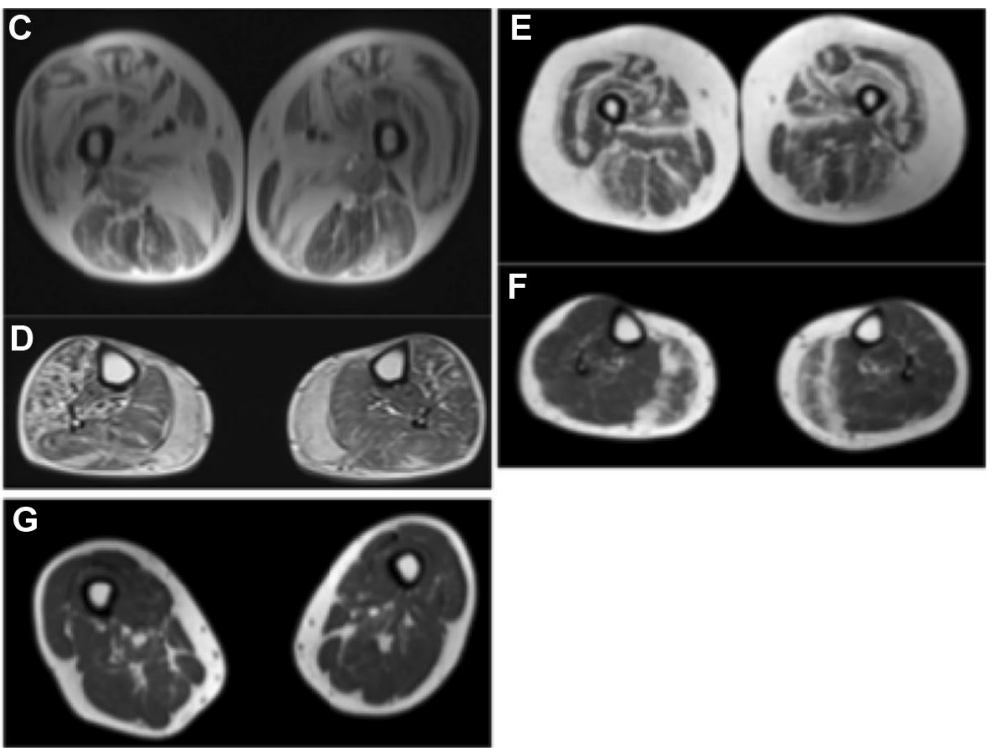

Fig. 2 Lower limb magnetic resonance imaging shows characteristic fatty degeneration in the center of the rectus femoris and a layered, "tigroid" pattern in the vastus lateralis of patients II-1 (a), II-2 (c),
II-3 (e), while thigh MRI was normal in the unaffected sibling II-4 (g) at 60 years of age. There is severe fatty replacement of the gastrocnemius medialis in all patients $(\mathbf{b}, \mathbf{d}, \mathbf{f})$
Fig. 3 Double staining using collagen VI (red) and merosin/ LAMA2 (green) antibodies in the normal control muscle (a) shows co-localization of the two labels, visualized as yellow labeling on the muscle fiber basal lamina. In a patient with early-onset severe collagenopathy (COL6A1), collagen (red) is largely detached from the basal lamina, resulting in green merosin labeling (b). The staining pattern in the proband's II-2 (c) and his sister's II-1 (d) muscle biopsies is not clearly abnormal, even though the interstitial space is enlarged showing collagen expression
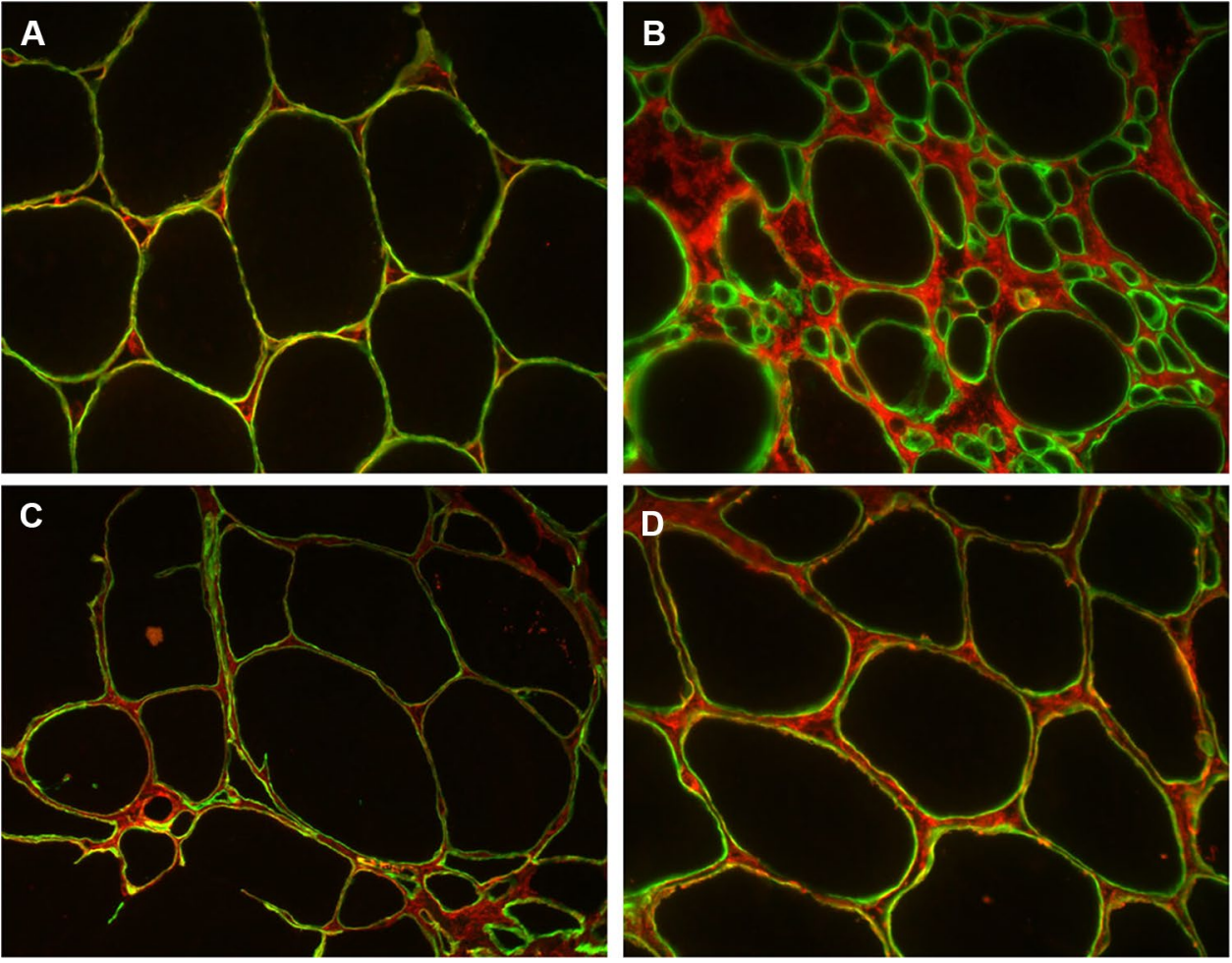

found to fully segregate with the phenotype in this family. Pathogenicity of the mutation is supported by the unusual and highly characteristic muscle MRI pattern, which is almost exclusively seen in collagenopathies [2], although it has also been rarely reported in calpainopathy [6]. Double immunofluorescence of collagen VI and merosin did not show obvious abnormalities, which is in line with previous reports in milder dominantly inherited collagenopathies, such as Bethlem myopathy. The double staining IF method is more useful in severe collagenopathy, where COL VI is 
clearly detached from the myocyte basal lamina, and the colocalization of COL VI and merosin is lost [2].

Collagen VI-related disorders are autosomal dominant or recessive disorders with phenotypes ranging from severe congenital muscular dystrophy to milder, adult-onset forms and are usually accompanied by variable degrees of joint contractures [2]. Our patients did not have contractures or spinal deformities, although some degree of Achilles tendon tightness was present in all. However, this is a nonspecific finding and not very significant considering the age of the patients.

LGMD-type of collagenopathy without contractures has been reported only rarely and according to the new ENMC classification, would be labeled as LGMD D5 (dominant) or LGMD R22 (recessive). The family reported here meets the criteria for a late-onset form of LGMD D5, caused by the COL6A2 mutation c. $2107 \mathrm{~A}>\mathrm{C}$ p.T703P.

Although sporadic inclusion body myositis is the most common muscle disease in patients over 50 years of age, it is important to recognize that several genetic myopathies and dystrophies may also manifest at this age. Indeed, some of the most common LGMD forms in Finland, such as LGMD2L (anoctaminopathy) and DNAJB6-related LGMD1D, frequently become symptomatic only after 50 years of age [7]. Accurate family history may be especially difficult to obtain in these very late-onset patients, because of the many other age-related neuromuscular complaints in this age group. Similarly, the proband (II-2) of our family had signs of neurogenic disease in the lower limbs both on EMG and muscle biopsy, which might have been considered the cause of his symptoms, had the muscle MRI not been performed. Our study, therefore, shows that muscle MRI is a valuable tool to help sorting out different neuromuscular disorders also in elderly patients.

Acknowledgements Open access funding provided by University of Turku (UTU) including Turku University Central Hospital.

\section{Compliance with ethical standards}

Conflicts of interest The authors declare that they have no conflicts of interest.

Ethical standards Informed consent and local ethics committee approval were obtained for this study.

Open Access This article is distributed under the terms of the Creative Commons Attribution 4.0 International License (http://creativeco mmons.org/licenses/by/4.0/), which permits unrestricted use, distribution, and reproduction in any medium, provided you give appropriate credit to the original author(s) and the source, provide a link to the Creative Commons license, and indicate if changes were made.

\section{References}

1. Straub V, Murphy A, Udd B, LGMD workshop study group (2018) 229th ENMC international workshop: Limb girdle muscular dystrophies-Nomenclature and reformed classification Naarden, the Netherlands, 17-19 March 2017. Neuromuscul Disord 28:702-710

2. Bönnemann C (2011) The collagen VI-related myopathies: muscle meets its matrix. Nat Rev Neurol 7:379-390

3. Scacheri PC, Gillanders EM, Subramony SH, Vedanarayanan V, Crowe CA, Thakore N et al (2002) Novel mutations in collagen VI genes: expansion of the Bethlem myopathy phenotype. Neurology 58:593-602

4. Evilä A, Arumilli M, Udd B, Hackman P (2016) Targeted nextgeneration sequencing assay for detection of mutations in primary myopathies. Neuromuscul Disord 26:7-15

5. Lampe AK, Flanigan KM, Bushby KM, Hicks D. Collagen Type VI-Related Disorders..In: Adam MP, Ardinger HH, Pagon RA, Wallace SE, Bean LJH, Stephens K, Amemiya A, editors. GeneReviews ${ }^{\circledR}$ [Internet]. Seattle (WA): University of Washington, Seattle; 1993-2019. 2004 Jun 25 [updated 2012 Aug 9].

6. Buendía J, Paipa A, Dominguez R et al (2016) Rolled cake sign in calpain related myopathies. Neuromuscul Disord 26:S100

7. Palmio J, Udd B (2014) Borderlines between sarcopenia and mild late-onset muscle disease. Front Aging Neurosci 6:267 